\title{
Chronic post-traumatic neuropathic pain of brachial plexus and upper limb: a new technique of peripheral nerve stimulation
}

\author{
Giorgio Stevanato • Grazia Devigili • Roberto Eleopra • \\ Pietro Fontana • Christian Lettieri - Chiara Baracco • \\ Franco Guida $\cdot$ Sara Rinaldo $\cdot$ Marzio Bevilacqua
}

Received: 4 April 2013 /Revised: 9 August 2013 / Accepted: 27 October 2013 / Published online: 21 February 2014

(C) The Author(s) 2014. This article is published with open access at Springerlink.com

\begin{abstract}
The aim of the study was to evaluate the effect on pain relief in patients with peripheral neuropathic pain after brachial plexus injuries using an implanted peripheral nerve stimulator applied directly to the nerve branch involved into the axillary cavity. Seven patients with post-traumatic brachial plexus lesions or distal peripheral nerve complaining of severe intractable chronic pain were enrolled in a single-centre, openlabel trial. Conventional drugs and traditional surgical treatment were not effective. Patients underwent careful neurological evaluation, pain questionnaires and quantitative sensory testing (QST). Surgical treatment consists of a new surgical technique: a quadripolar electrode lead was placed directly on the sensory peripheral branch of the main nerve involved, proximally to the site of lesion, into the axillary cavity. To assess the effect, we performed a complete neuroalgological evaluation and QST battery after 1 week and again after 1, 6 and 12 weeks. All patients at baseline experienced severe pain with severe positive phenomena in the median (5) and/or radial (2) territory. After turning on the neuro-stimulator system, all patients experienced pain relief within a few minutes ( $>75 \%$ and $>95 \%$ in most), with long-lasting pain relief with a reduction in mean Numerical Rating Scale (NRS) of $76.2 \%$
\end{abstract}

\footnotetext{
G. Stevanato $\cdot$ F. Guida

Neurosurgery Unit, Neuroscience Department, "Ospedale dell'Angelo", General Hospital, Mestre-Venice, Italy

G. Devigili $(\varangle) \cdot$ R. Eleopra $\cdot$ C. Lettieri $\cdot$ S. Rinaldo

Neurological Unit, Neuroscience Department, University Hospital

"S. Maria della Misericordia", Udine, Italy

e-mail: devigili.grazia@aoud.sanita.fvg.it

P. Fontana $\cdot$ C. Baracco

Pain Medicine, "Ospedale dell'Angelo", General Hospital,

Mestre-Venice, Italy

M. Bevilacqua

Pain Management Unit, Anesthesia Department, "Ospedale S. Maria

di Ca' Foncello", General Hospital, Treviso, Italy
}

after 6 months and of $71.5 \%$ after 12 months. No significant adverse events occurred. We recommend and encourage this surgical technique for safety reasons; complications such as dislocation of electrocatheters are avoided. The peripheral nerve stimulation is effective and in severe neuropathic pain after post-traumatic nerve injuries of the upper limbs.

Keywords Peripheral nerve stimulation (PNS) · Traumatic brachial plexus injuries $\cdot$ Neuropathic pain $\cdot$ Quantitative sensory testing (QST)

\section{Introduction}

Chronic peripheral neuropathic pain due to peripheral nerve injury often results in significant suffering and impaired quality of life. It is poorly responsive to drugs usually used for the treatment of neuropathic pain (NP) such as anticonvulsants and membrane stabilizers and opioids, even at high dosages [25]. For patients who have no response to medications and high rate of deafferentation (i.e. root avulsion), the ablative dorsal root entry zone (DREZ) lesion procedure is traditionally considered, while spinal cord stimulation (SCS) has been reported as a conservative technique in particular in case of prevalent central mechanisms like in CRPS or root avulsion $[14,18]$.

Therefore, an effective treatment for neuropathic pain still remains a major clinical challenge. Peripheral nerve stimulation (PNS) has been used by a small group of neurosurgeons for the treatment of chronic peripheral neuropathic pain since 1967 [30]. Although in literature, PNS was demonstrated to be quite successful in the short and medium term [4, 6, 9, 21, 27, 29], PNS has never become a standard technique for treatment of NP syndromes. This may be due to technical difficulties with paddle-type electrodes inserted around the peripheral nerve and to the risk of infections or electrode dislocation. 
The mechanism by which PNS produces analgesia is still unclear. One of the possible mechanisms is based on the assumption that direct application of low-intensity, highfrequency electrical current onto a peripheral nerve can elicit the A-beta myelinated fibres and produce analgesia according to the "gate-control" mechanism [16]. Another mechanism proposed was the ortho- and antidromic collision in $A \beta$ fibres $[10,20]$. Both mechanisms as a key part of PNS are closely related to the brain function and brain plasticity in particular $[22,26]$.

Thus, we hypothesized that the implantation of PNS leads directly to the nerve branch mainly involved in the painful syndrome, proximally to the site of injury, at the brachial plexus may produce analgesia or significant pain relief in patients with traumatic nerve injuries. For this purpose, a pathway that is at least partially preserved is required.

In this study, we assessed the short- and medium-term outcomes of PNS in a number of patients with peripheral post-traumatic neuropathic pain in the upper arm by using a new and innovative surgical technique. The aim of the study was to evaluate pain relief in this carefully selected group of patients due the direct stimulation of the nerve branches administered by means of lead inserted with an original surgical technique.

\section{Methods}

\section{Patients}

Patients affected by intractable pain due to peripheral nerve injuries and referred to our neurological unit for advanced pain treatment during the years 2007-2010 were considered for PNS. We selected only patients with sensory sensation preserved in the painful skin area. Patients with root avulsion or spinal cord lesions were excluded.

A detailed medical history and neurological examination were collected. Peripheral neuropathic pain was defined as chronic pain in an area of sensory abnormality corresponding to the nerve lesion and with an onset less than 6 months after the lesion, according to the grading system proposed by the NeuPSIG guideline [7, 28].

We decided to include patients with pain defined as "intractable" for no response to the pharmacological and traditional surgical treatment and "chronic" if present from at least 1 year without significant changes in intensity and characteristics. None of the patients underwent microsurgical DREZotomy.

Patients were carefully selected for PNS after an extensive baseline assessment. Therefore, we identify the following criteria for inclusion: (1) clear identification of an isolated injured nerve (i.e. selective branch of brachial plexus, median nerve, radial nerve) by means of clinical and electroneurographic and electromyographic evaluation as the unique cause of pain; (2) complete although transient pain relief following a diagnostic nerve block with local anaesthetics; (4) poor response ( $<50 \%$ of pain relief) to all other treatments after a trial period of at least 1 year, including medication use and surgical treatment of the injury site such as neurolytic procedures, neuroma resection, nerve grafting and transection; (4) absence of major psychiatric disorders, such as personality disorders or major depression, as assessed by psychological examination using Minnesota Multiphasic Personality Inventory (MMPI-2); (5) signing of a written informed consent for the study and for the surgical procedure.

The study was single centre and open label and received institutional review board approval.

Neurological and algological baseline examination

All subjects underwent a complete neurological and algological examination of the upper and lower trunk, medial, lateral and posterior cords of the brachial plexus and peripheral nerves, in order to assess the motor and sensory loss distribution and positive phenomena described below.

Superficial and pinprick sensations were examined in order to identify negative sensory signs (sensory loss) and positive sensory signs (evoked and spontaneous pain, paraesthesias). The neurological examination was performed using cotton gauze (light touch and dynamic mechanic allodynia test) and a brush, disposable safety needle (hypoalgesia, pinprick hyperalgesia, after-sensation test), repetitive pinprick (2 Hz for $30 \mathrm{~s}$ ) and glass vials filled with cold and hot water (thermal sensation, allodynia test, after-sensation test). Deep tendon reflexes were classified as normal, decreased (if present with reinforcement) or absent. All muscle groups of the upper arm were evaluated, and muscle strength was graded using the Medical Research Council (MRC) score. Intensity of spontaneous pain, allodynia including static mechanical (pressure), dynamic mechanical (brush), heat or cold (thermal) and hyperalgesia were graded using the Numerical Rating Scale (NRS) (0-10) scale. We used the following descriptors for quality of pain: throbbing, lancinating, unpredictable, lightning-like, sharp, shooting, aching, burning, scalding, pruritic [5, 7]. Pain attacks were described in terms of intensity, duration and frequency.

We collected a picture for the pain on the skin and a detailed pain map and sensory abnormality distribution on a body chart.

Finally, the clinical evaluation was completed with upper arm examination in order to rule out any intrinsic joint disease and acromioclavicular pathology, and in patients with lower brachial plexus involvement the elevated arm stress test $[20,23]$ was performed in order to relieve thoracic outlet syndrome [24]. 
Quantitative sensory testing

Thresholds for cold and warm sensation (CS, WS) and cold and heat pain (CP, HP) were obtained bilaterally on the thenar and hypothenar eminences and on other sites according with the neuroanatomical distribution of pain and negative signs, with a $30 \times 30 \mathrm{~mm}$ thermode of the TSA-II Neuro Sensory Analyzer (Medoc, Israel). Method of limits has been performed. Each test was repeated four times for each side, and the perceived threshold was defined as the average of peak temperatures. Subjects were instructed to push the response button, which recorded the temperature and reset the thermode back to baseline, as soon as they detected a change in temperature for CS and WS, or only if the sensation changed from cold or hot to painful sensation.

\section{Surgical procedure}

Surgical procedures were performed at the General Hospital of Mestre-Venice between 2007 and 2010. All implantations were performed under general anaesthesia and under strict sterile conditions by one surgeon (G.S.). The nerves selected for surgery were exposed using the technique of brachial plexus exposure by the axillary approach. Patients were supine with the upper arm abducted by $90^{\circ}$, all the nerve and vascular structures were exposed, identified and classified as shown in Fig. 1.

Two on-point leads (Medtronic Inc., Minneapolis) were used for the stimulation of the radial or/and the median nerve, respectively. Each lead has four platinum-iridium electrodes on a silicone-rubber mesh. During the surgical procedure, the leads were placed upon the sensory portion of the nerve according to the Sunderland's scheme [26] about $7 \mathrm{~cm}$ from the nerve origin, on the postero-medial aspect of median nerve and about $5 \mathrm{~cm}$ from the antero-medial part of the rising radial nerve and carefully anchored using the paddle lead mesh (Fig. 1C) with no absorbable sutures in order to avoid nerve compression. This technical solution allows nerve protection by the surrounding fibrosis and provides stability of the overall system.

If the patient had motor function preserved at baseline, a stimulation trial was carried out by means of NeuroPulse ${ }^{\mathrm{TM}}$ (Bovie Medical Corporation) to confirm the correct position on sensory nerve fibres in order to avoid the stimulation of motor fibres. In three patients (1-3), the on-point leads were placed proximally to the site of lesion; in the other four patients (4-7), the on-point leads were placed distally at the rise of sensitive nerve involved in painful syndrome, after verifying the partial integrity of somatosensory signals. The electrodes were tunnelled under the skin, making several loops to minimize traction or dislocation with the upper arm movement, and then connected to the temporary extensions.
During the trial period, an external stimulator (ENS, Medtronic Inc, Minneapolis) was used for a mean of 7 days.

After the trial period, temporary extensions were removed and replaced by permanent extensions which were then connected to an implantable pulse generator (IPG, mod.Prime advanced, Medtronic Inc., Minneapolis) that was secured in the subclavicular subcutaneous pocket.

During the first trial, about $24 \mathrm{~h}$ after the procedure, the pulse rate, width and voltage that produced the best response were selected. Stimulator setting was with width and pulse rate fixed for each patient. All patients were set for 24-h stimulation.

Daily pain evaluation and scales

Patients were requested to evaluate their pain intensity with the use of an 11-point Likert scale (NRS), where " 0 " indicates "no pain" and "10" indicates "the worst imaginable pain". Additionally, they were asked to describe their pain with the use of the pain questionnaire $[6,19]$.

Pain and clinical follow-up evaluation

Patients were assessed during scheduled follow-up visits for efficacy evaluations after 1 week, 1 month, 6 months and 1 year from the IPG implantation. Evaluation of global pain relief was graded on a percentage scale of four categories: 0-24\% (poor), 25-49\% (fair), 50-74\% (good) and 75-99\% (excellent). Patients underwent NRS scales and pain questionnaires. Finally, to quantify the pain-positive phenomena such as thermal hyperalgesia and allodynia, quantitative sensory testing (QST) was assessed at baseline, 1 month and 12 months.

\section{Statistics}

A two-tailed $t$ test was used to compare NRS values and analgesic consumption prior to surgery and during the follow-up. Results at the $P<0.05$ level were regarded as statistically significant.

\section{Results}

We identified seven patients, all men, fulfilling the inclusion criteria and suitable for PNS. All patients had a post-traumatic brachial plexus lesion or distal peripheral nerve, complaining of severe intractable pain characterized by allodynia, paradoxical pain and ongoing pain. The clinical data are reported in Tables 1 and 2. In four patients, a traumatic peripheral nerve lesion occurred, while in three, a traumatic brachial plexus postganglionic injury caused the peripheral NP syndrome. The patients' ages ranged from 17 to 68 , with a median age of 46. Pain duration prior to PNS ranged from 19 months to 
Fig. 1 Surgical procedure. The nerves selected for surgery were exposed using the technique of brachial plexus exposure by the axillary approach $(\mathbf{a}-\mathbf{b})$. The leads were placed upon the sensitive portion of the nerve according to Sunderland's scheme about $4 \mathrm{~cm}$ far from the rising nerve, on the lateral aspect of the median nerve (c) and carefully anchored by means of lead paddle mesh (d) with no absorbable sutures in order to avoid nerve compression. X-ray studies (e-f) show the position of the leads and IPG with different position of the upper arm
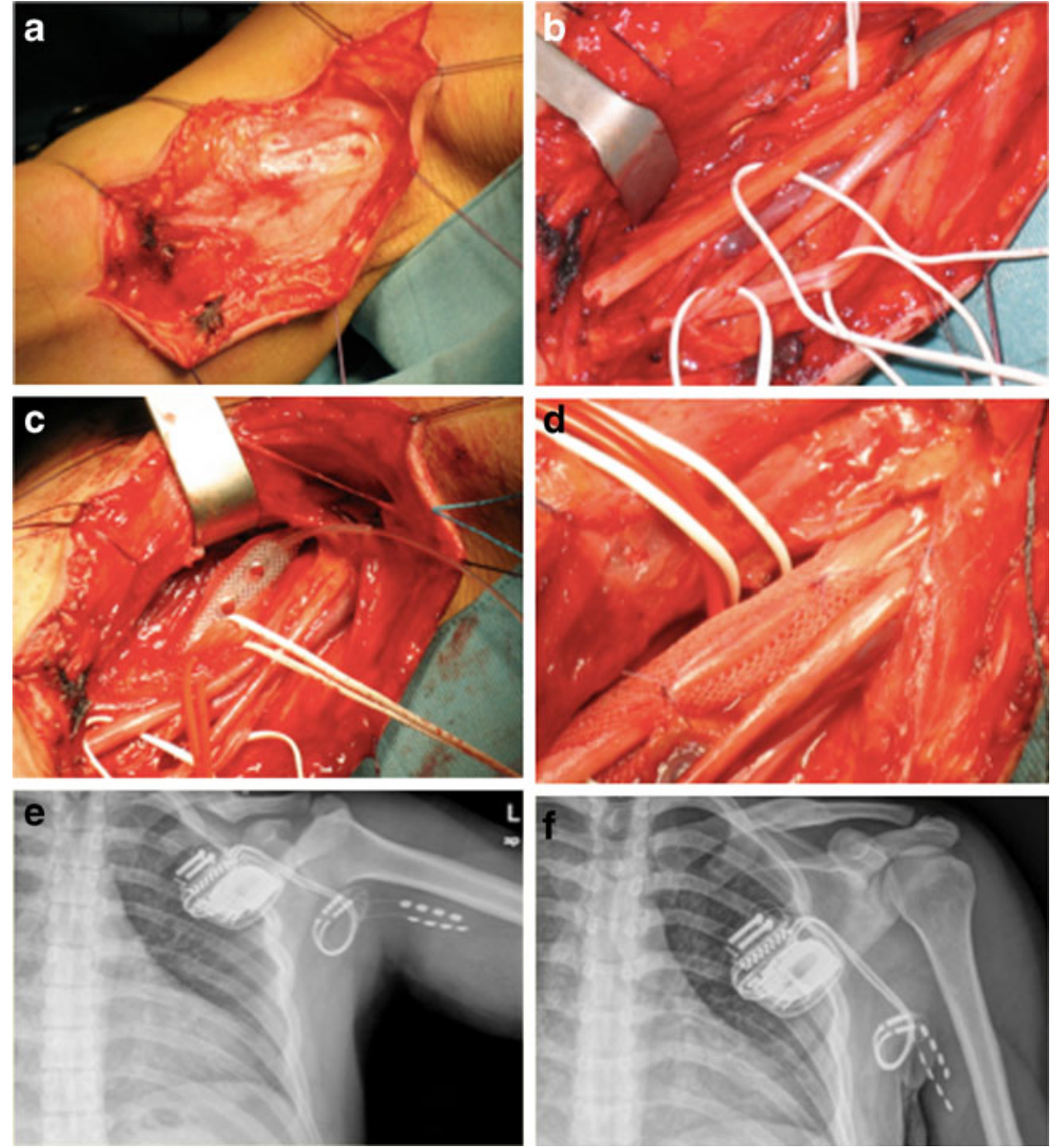

31 years. The mean baseline NRS was 9/10, indicating moderate to severe pain intensity before surgery. The patients described the painful syndrome with several qualities, including cutting sensation, burning sensation and constriction with impressive positive phenomena like electric shock after light touch or movement, several times each day. From the history, all patients had previously received neurosurgical treatments including neurolysis. Therefore, all patients were prescribed conventional medications (analgesics, antidepressants, anticonvulsants) (see Table 2) and repeated nerve blocks. None of the patients met the research diagnostic criteria for complex regional pain syndrome II (CRPS II) [8].

The results based on pain relief were classified as "good" in all patients. The stimulation parameters of PNS were similar in all patients, with a rate pulse of $50 \mathrm{~Hz}$, a width of $250 \mu \mathrm{s}$ and an amplitude ranging from 0.15 to $0.30 \mathrm{~V}$. This stimulation setting the PNS does not produce any sensation like paresthesia in the skin area.

Overall, pain intensity decreased from an NRS of $9 \pm 1.15$ before surgery to $2.14 \pm 1.57$ at the6-month follow-up and to $2.57 \pm 1.13$ at the 12-month follow-up $(P<0.001)$ (Fig. 2). No complications like infections, dislocation or electrodes dislocations or migrations occurred in any of the patients.
QST battery at baseline and at follow-up

Thermal thresholds were abnormal in all patients according to the neuroanatomical distribution of negative signs as expected by the sensory clinical evaluation; in particular, we found higher cold and warm sensation thresholds in selective skin areas. In six of seven patients at baseline, we found positive phenomena characterized by cold or warm allodynia and in five wrong painful sensation after cold and warm stimuli into the non-painful range. The abnormal painful sensation was reported as an electric shock or burning sensation with wide skin area distribution, higher than expected by the nerve injured distribution, suggesting peripheral or central hypersensitivity phenomena.

After 6 months, at the follow-up evaluation, thermal QST evaluation showed unchanged negative sign pattern and attenuation or complete resolution of positive phenomena (Fig. 2).

\section{Discussion}

Peripheral nerve stimulation (PNS) is a neuro-modulation technique in which electrical current is applied to the 
peripheral nerves to improve chronic pain. It was first described by Wall and Sweet in 1967 [30]; they used an electrode to stimulate a peripheral nerve in post-traumatic neuralgic pain. A variety of techniques have since been developed [20]. In the 1970s, PNS was seldom performed because the high morbidity and poor long-term outcomes related to poor patient selection and technical limitations for inadequate devices. However, growing evidences suggested that PNS is effective, in particular NP syndromes characterized by peripheral nerve lesions or irritation with pain or positive phenomena with a localized peripheral nerve distribution [9, 21, 27, 31, 32].

In this study, we show a selected case series of patients complaining of severe neuropathic pain involving the upper arm with pain phenomena extremely consistent to a peripheral nerve distribution. This clinical picture is usually at high risk for non-responsiveness with other neuro-modulation techniques such as spinal cord stimulation (SCS) or motor cortex stimulation (MCS) $[1,3,20]$. In particular, SCS is reported to be more effective in painful syndrome with spinal hyperactivity in the dorsal part of spinal cord but compared with PNS is less selective in covering the painful skin area of peripheral nerve injuries and has higher risk of dislocation $[13,26]$. MCS in peripheral neuropathic pain has been applied in few case series [15] and has unpredictable antalgic effect to be compared with the other techniques.

PNS pain relief can be explained by the "gate-control" theory of pain [16]; the peripheral nerve stimulation produces direct electrical effects by recruitment of primary afferent Abeta and A-delta fibres that project at the spinothalamic tract and dorsal columns and A-alpha fibres that cause segmental inhibition through presynaptic inhibitory interneurons. These electrical effects seem to be more selective and effective than SCS.

However, peripheral nerve stimulation through a percutaneous approach $[11,17]$ — in particular, for upper and lower limb - has a less favourable chronic outcome and higher morbidity due to displacement-related movement of adjacent structures (i.e. tendons, nerves, vascular structures). These dynamic forces can affect distraction and translation of the electrodes from their associated nerves. Otherwise, for similar reasons, the migration of electrodes is one of the most frequent complications, occurring in up to $33 \%$ of cases [12], and requires revision. For these reasons, we propose this new surgical technique with the allocation of on-point lead into soft tissue of the armpit, without tendon-muscular structure, which avoids the nerve compression [26]. Notably, this technique minimizes the chance of electrode dislocation, is selective on the target nerve branches and allows a low-intensity stimulation that saves battery life.

In our opinion, the success of PNS implantation in all our cases is based on a careful patient selection process. We applied selection criteria based on literature review $[9,26]$ and our clinical and neurophysiological experience in 
Table 2 Painful syndrome phenotype and neuroalgological pattern

\begin{tabular}{|c|c|c|c|c|c|c|c|c|c|}
\hline \multirow[t]{2}{*}{ Patient no. } & \multirow{2}{*}{$\begin{array}{l}\text { Background pain } \\
\text { Intensity, NRS }\end{array}$} & \multicolumn{3}{|c|}{ Spontaneous pain paroxysms } & \multicolumn{5}{|c|}{ Evoked pain, 0-10 NRS } \\
\hline & & Intensity, NRS & Duration (s) & Frequency & Brush & Pinprick & Repetitive pinprick & Cold & Warm \\
\hline 1 & 10 & 7 & 15 & 4/day & 10 & 8 & 5 & 5 & 8 \\
\hline 2 & 8 & 5 & 30 & $1 / \mathrm{h}$ & 9 & 5 & 4 & 3 & 2 \\
\hline 3 & 10 & 10 & 10 & $1 / \mathrm{h}$ & 10 & 1 & 6 & 6 & 9 \\
\hline 4 & 7 & 8 & 160 & $10 / \mathrm{h}$ & 10 & 10 & 10 & 10 & 7 \\
\hline 5 & 9 & 9 & 5 & $1 /$ month & 7 & 7 & 8 & 5 & 8 \\
\hline 6 & 10 & 10 & $30-45$ & $1 / \mathrm{h}$ & 5 & 8 & 10 & 7 & 6 \\
\hline 7 & 9 & 5 & $2-3$ & 3/day & 6 & 6 & 5 & 5 & 6 \\
\hline
\end{tabular}

neuropathic pain studies (the details of which are included in the paragraph on Methods). We improve the sensory profile evaluation using a semiquantitative instrument such as a quantitative sensory testing battery, which is particularly useful for quantifying positive and negative signs at baseline and after treatment [2].

In literature, the use of a prognostic nerve anaesthetic block prior to PNS has been suggested by several groups [13, 29], and from common practice it is well accepted that failure to respond to a nerve block is a poor prognostic factor for PNS. For that reason, complete although temporary pain relief following a diagnostic nerve block with local anaesthetics was required as a pre-test in our screening evaluation. The use of the local anaesthetic injection may help us to confirm the selection of the nerve target and identify the prevailing peripheral source of the particular pain syndrome.
As reported also by other groups [29], we observe that PNS modified neither heat nor cold thresholds assessed by the QST battery. Therefore, the main finding for the QST battery was the clear anti-allodynic or anti-hyperalgesic effect. The improvement of positive phenomena is a direct effect of the PNS stimulation, while the negative signs in our patients are the consequence of the nerve lesion, and PNS is obviously ineffective in improving a loss of peripheral nerve function such as sensory sensation. However, PNS can affect changes in nociceptive signalling that modulate peripheral and central sensitization leading to a reduction of allodynia.

The 1-year follow-up confirms the long-lasting beneficial effect of SCS, nearly unchanged between visit after 1 month and visit after 12 months.

Finally, we assessed the usefulness of thermal QST to detect the positive phenomena in our patients, with a sort of
Fig. 2 Average pain intensity scores before and after PNS implantation during a follow-up period of 1 year

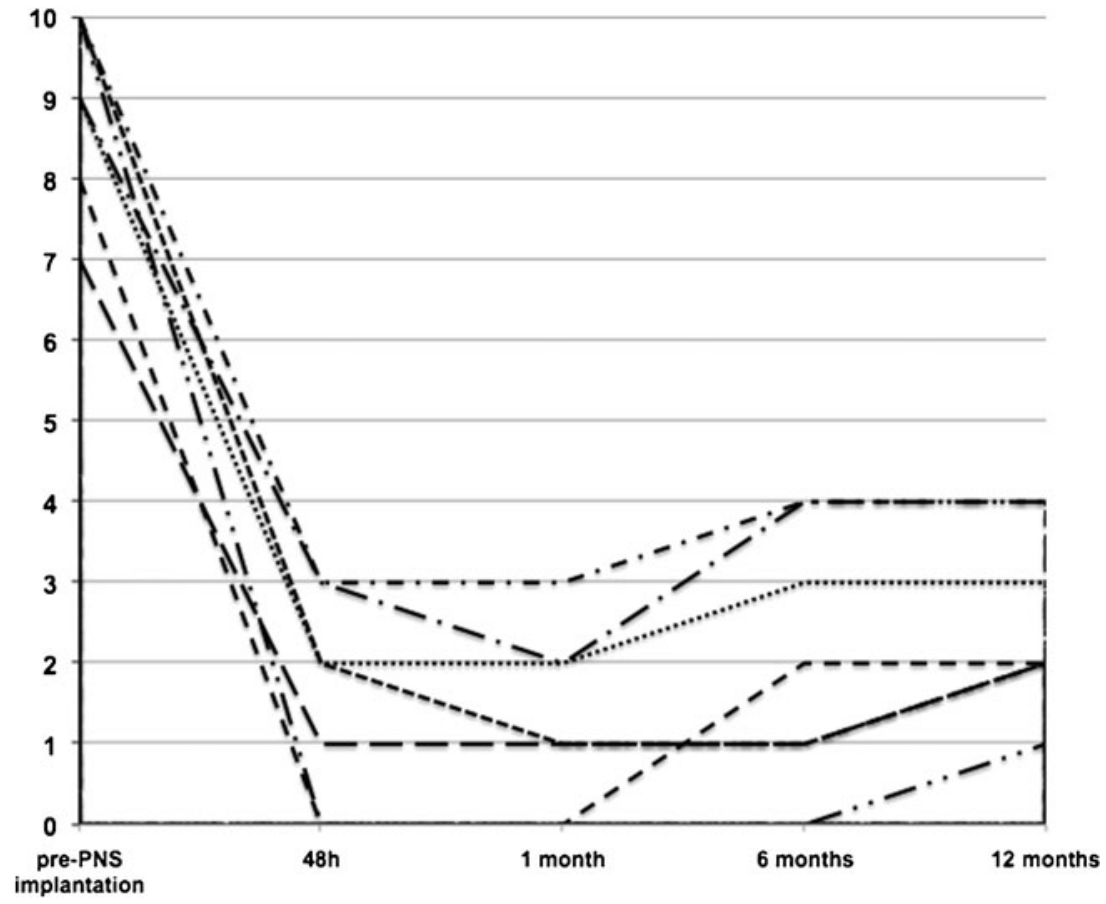


stereotypical and reliable QST pattern also confirmed by several observations (data not shown) and, above all, for follow-up evaluation to clearly detect the anti-allodynic effect of PNS.

\section{Conclusions}

This new PNS technique of electrode implantation can result in significant pain relief in carefully selected patients with peripheral neuropathic pain due to post-traumatic nerve injuries, reducing the complication rate and preserved neuroanatomical structures. It should therefore be considered as a reasonable treatment of patients suffering from otherwise intractable painful neuropathies of the upper arm.

Disclosure The authors report no conflict of interest concerning the materials or methods used in this study or the findings specified in this paper.

Open Access This article is distributed under the terms of the Creative Commons Attribution License which permits any use, distribution, and reproduction in any medium, provided the original author(s) and the source are credited.

\section{Reference}

1. Bennet MI, Tai YM (1997) Cervical dorsal column stimulation relieves pain of brachial plexus avulsion. J Royal Soc Med 87:5-6

2. Cruccu G, Sommer C, Anand P, Attal N et al (2010) EFNS guideline on neuropathic pain assessment: revised 2009. Eur J Neurol 17:1010-1018

3. van Dongen R, Cohen SP, van Kleef M et al (2011) Traumatic plexus lesion. Pain Practice 11(4):414-420

4. Eisenberg E, Waisbrod H, Gerbershagen HU (2004) Long-term peripheral nerve stimulation for painful nerve injuries. Clin J Pain 20(3):143-6

5. Galer BS, Jensen MP (1997) Development and preliminary validation of a pain measure specific to neuropathic pain: the Neuropathic Pain Scale. Neurology 48(2):332-8

6. Goroszeniuk T, Kothari SC, Hamann WC (2007) Percutaneous implantation of a brachial plexus electrode for management of pain syndrome caused by a traction injury. Neuromodulation 10:148-55

7. Haanpää M, Attal N, Backonja M, Baron R, Bennett M et al (2011) NeuPSIG guidelines on neuropathic pain assessment. Pain 152(1):14-27

8. Harden RN, Bruehl SP (2006) Diagnosis of complex regional pain syndrome: signs, symptoms, and new empirically derived diagnostic criteria. Clin J Pain 22(5):415-9

9. Hassenbusch SJ, Stanton-Hicks M, Schoppa D, Walsh JG, Covington EC (1996) Long-term results of peripheral nerve stimulation for reflex sympathetic dystrophy. J Neurosurg 84(3):415-23

10. van den Honert $C$ et al (1979) The response of the myelinated nerve fiber to short duration biphasic stimulation currents. Ann Biomed Eng 7(2):117-25

11. Huntoon MA, Burgher AH (2009) Ultrasound-guided permanent implantation of peripheral nerve stimulation (PNS) system for neuropathic pain of the extremities: original cases and outcomes. Pain Med 10(8):1369-77
12. Ishizuka K, Oaklander AL, Chiocca EA (2007) A retrospective analysis of reasons for reoperation following initially successful peripheral nerve stimulation. J Neurosurg 106:388-390

13. Kozak J, Kobesova A, Vrba I, Steindler J, Kolar P (2011) Peripheral nerve stimulation in intractable neuropathic pain. Neuroendocrinol Lett 32(3):226-233

14. Lai H-Y, Lee C-H, Lee S-T (2009) High cervical spinal cord stimulation after failed dorsal root entry zone surgery for brachial plexus avulsion pain. Surg Neurol 72:286-289

15. Lefaucheur J-P, Drouot X, Cunin P, Bruckert R, Lepetit H et al (2009) Motor cortex stimulation for the treatment of refractory peripheral neuropathic pain. Brain 132:1463-1471

16. Melzack R, Wall PD (1965) Pain mechanisms: a new theory. Science 150:971-979

17. Mobbs RJ, Nair S, Blum P (2007) Peripheral nerve stimulation for the treatment of chronic pain. J Clin Neurosci 14(3):216-21

18. Monti E (2004) Peripheral nerve stimulation: a percutaneous minimally invasive approach. Neuromodulation 7(3):193-6

19. Padua L, Briani C, Jann S, Nobile-Orazio E et al (2009) Validation of the Italian version of the Neuropathic Pain Symptom Inventory in peripheral nervous system diseases. Neurol Sci 30(2):99-106

20. Piva B, Shaladi A, Saltari R, Gilli G (2003) Spinal cord stimulation in the management of pain from brachial plexus avulsion. Neuromodulation 6(1):27-31

21. Reverberi C, Bonezzi C, Demartini L (2009) Peripheral subcutaneous neurostimulation in the management of neuropathic pain: five case reports. Neuromodulation 2:146-155

22. Ristić D, Spangenberg P, Ellrich J (2008) Analgesic and antinociceptive effects of peripheral nerve neurostimulation in an advanced human experimental model. Eur J Pain 12(4):480-90

23. Roos DB (1966) Transaxillary approach for the first rib resection to relieve thoracic outlet syndrome. Ann Surg 16:354-8

24. Schwartzman RJ, Grothusen JR (2008) Brachial plexus traction injury: quantification of sensory abnormalities. Pain Med 9(7):950-7

25. Sindrup SH (2000) Pharmacologic treatment of pain. Neurology 55: 915-920

26. Stanton-Hicks M, Panourias IG, Sakas DE, Slavin KV (2011) The future of peripheral nerve stimulation. Prog Neurol Surg 24:210-7

27. Sunderland SS (1978) The brachial plexus. Normal anatomy. In: Sunderland SS (ed) Nerves and nerve injuries. Churchill Livingstone, Edinburgh, pp 854-869

28. Treede RD, Jensen TS, Campbell JN, Cruccu G, Dostrovsky JO et al (2008) Redefinition of neuropathic pain and a grading system for clinical use: consensus statement on clinical and research diagnostic criteria. Neurology 70:1630-5

29. Van Calenbergh F, Gybels J, Van Laere K, Dupont P, Plaghki L, Depreitere B, Kupers R (2009) Long term clinical outcome of peripheral nerve stimulation in patients with chronic peripheral neuropathic pain. Surg Neurol 72(4):330-5

30. Wall PD, Sweet WH (1967) Temporary abolition of pain in man. Science 155(3758):108-9

31. Weiner RL, Reed KL (1999) Peripheral neurostimulation for control of intractable occipital neuralgia. Neuromodulation 2(3):21721

32. Weiner RL (2003) Peripheral nerve neurostimulation. Neurosurg Clin N Am 14(3):401-8

\section{Comments}

Stefano Ferraresi, Rovigo, Italy

The paper written by Dr. Stevanato \& colleagues from Mestre has the great merit to focus on a big therapeutic challenge for peripheral nerve surgeons and algologists. 
As a matter of fact, the neuropathic pain following non-avulsive severe nerve injuries of the various kinds may be truly intractable.

The DREZ microlesion is theoretically incorrect and practically ineffective, so as other invasive procedures carried out directly on the nerve, namely neurolysis, wrapping or, worst of all, undue section of the involved nerve(s) which herald dreadful long-term complications.

These patients are often ping-ponged back and forth between various pain therapists and normally sedated with opioids, anticonvulsants, antidepressants and, more recently, cannabinoids.

Very occasionally, a few of them show some improvement, but the vast majority of them declare themselves unsatisfied.

PNS stimulation, also in our hands, is the procedure more effective than any other and, most of all, is fully reversible. This warrants that, in case of failure, there is no risk of a permanent worsening effect.

At a very long-term follow-up, however, these patients are unwilling to accept this method of cure, this being due to a sort of "addiction" to the neuromodulation effect and to a psychological rejection of its "robotic" aspect, brought by the presence of the subcutaneous devices.

Many patients, at the end, give up stimulation and, last but not least, ask for removal of the device.

More experience is needed in multiple nerve injuries (all the patients here presented had a median nerve neuropathic pain), but a more accurate consensus on the long-term psychological aspects is also desirable. This, especially, at the light of the exceedingly high costs of the procedure is not fully compatible with the spending review program of our NHS (National Health System).
Miran Skrap, Udine, Italy

The management of neuropathic pain is still a challenge because the response to most drugs remains unpredictable or poor. In particular, it is true for post-traumatic pain. The authors present their experience about seven patients who complained severe neuropathic pain due to a posttraumatic lesion of the brachial plexus. They described that their technique of implanting leads to the proximal part of the peripheral nerve which has been identified by intraoperative stimulation. The on-point lead has been placed at the level of the sensory portion of the nerve.

The peripheral nerve stimulation is a well-known technique described more than 50 years ago. Since then, a variety of techniques of implantation and devices have been developed. In recent years, thanks to improved devices, the use of PNS has increased.

The peculiarity of this technique is the accuracy of the intraoperative selection and the stimulation of the sensory nerve fibres involved in the pain syndrome. Even the importance of axillary approach is highlighted which should minimize the risk of dislocation of the nerves.

Furthermore, these good results in the pain relief rate can be achieved with a careful patient selection by clinical, pain and sensory profile evaluation which allows to identify the specific nervous target. Furthermore, the low intensity of stimulation required to induce pain relief is below the sensory threshold.

A longer follow-up period to confirm this data is anyway needed, but this neurophysiological/neurosurgical approach may offer future suggestions in the treatment of post-traumatic peripheral neuropathic pain. 